\title{
Prediction of Complication Development After Kidney Transplantation Using Blood Plasma Redox Potential Monitoring
}

Dol: $10.17691 / \mathrm{stm} 2015.7 .4 .11$

Reeeived August 12, 2015

V.A. Kolesnikov, DSc, Professor, Rector';

A.K. Evseyev, PhD, Senior Researcher, Laboratory of Cellular and Physicochemical Medical Technologies;

A.N. Elkov, PhD, Senior Researcher, Department of Treatment of Acute Poisoning;

A.V. Pinchuk, MD, PhD, Associate Professor, Head of the Department of Kidney and Pancreas Transplantation';

L.S. Kokov, MD, DSc, Professor, Corresponding Member of Russian Academy of Sciences, Head of the Department of Rentgenosurgical Methods of Diagnosing and Treatment';

T.G. Tsarkova, PhD, Professor, Department of the Technology of Electrochemical Processes';

M.M. Goldin, DSc, Professor, Leading Researcher, Laboratory of Cellular and Physicochemical Medical Technologies²

'D. Mendeleyev University of Chemical Technology of Russia, 9 Miusskaya Square, Moscow, 125047, Russian Federation; ${ }^{2}$ N.V. Sklifosovsky Research Institute of Emergency Care, 3 Bolshaya Sukharevskaya Square, Moscow, 129010,

Russian Federation period.

A problem of great concern in transplantology is inability of reliable prediction of the initial function of the graft in the early postoperative

The aim of the investigation was to assess reliability of predicting complications in the early postoperative period after kidney transplantation on the basis of the data obtained by monitoring redox potential (RP) in blood plasma.

Materials and Methods. 60 patients were examined after kidney transplantation in the early postoperative period in N.V. Sklifosofsky Research Institute of Emergency Care (Moscow). Two groups were formed: group 1 without complications in the postoperative period ( $n=36$ ) and group 2 with complications $(n=24)$. A total of 982 analyses were performed. Graft condition was evaluated basing on clinical observations, laboratory findings, US examination and needle graft biopsy. Electrochemical measurements were performed using platinum microelectrode. Experimental data were analyzed by the software packages Statistica 6.0 (StatSoft), EViews 8.0 (IHS Global, Inc.) and Visual Basic 6.0 IDI (Microsoft).

Results. Prediction was made by assessing the probability of complication development as a function of statistical characteristics of clinical and laboratory parameters. Comparison of the mean RP values dynamics in the examined groups in the course of monitoring showed, that the difference between them has reached $12 \mathrm{mV}$ already by day 5 , and $18 \mathrm{mV}$ by day 10 . And even on day 25 the difference in the mean RP values remains not less than $10 \mathrm{mV}$. To assess the dependence of complication occurrence probability on the RP value in blood plasma probit analysis, which has been previously used only in toxicology, was applied. The normal character of distribution was proved with the help of five criteria of testing for symmetry and for excess value. The application of probit analysis enabled us to assess the probability of complication development depending on the RP value on a selected day of the postoperative period during the patient's hospital stay. kidney.

Thus, application of RP monitoring proved to give a high probability of early prediction of complications in patients with transplanted

Key words: kidney transplantation; complications after transplantation; redox potential; probit analysis.

Complications in the early postoperative period after organ transplantation are diagnosed and evaluated by analyzing the whole complex of clinical and laboratory data on the condition of various body systems. Currently, methods enabling one to get information on functioning of one of the body systems, redox (oxidation-reduction)

For contacts: Goldin Mark Mikhailovich, e-mail: markmgold@gmail.com 
system, in particular, in terms, for example, of the prooxidant and antioxidant balance in the organism, are of great interest [1]. Impairment of bodily systems work due to some pathological processes may lead to both the elevation of prooxidant level (at least to the oxidative stress) and to the inhibition of the natural oxidative processes. The fact, that disturbance in the redox system is not of the local character, but affects functioning of the whole organism, draws special attention. It is also important, that assessment of the redox system condition may be conducted using simple methods of bodily liquid analysis (blood, blood plasma and so on).

Early diagnosis of the prooxidant and antioxidant balance can be performed by electrochemically measuring open-circuit platinum electrode potential in blood plasma, or the so called redox potential (RP) [2]. The measured value of the platinum electrode potential in patient's blood plasma is estimated to be an integral indicator of the redox balance [3]. Besides, it was found, that monitoring of RP value may be used not only for diagnosis, but for the prognostic purpose as well (owing to the sensitivity of the body redox system), as it helps to reveal signs of dysfunction several days before the condition is confirmed by clinical and laboratory findings [4].

Dysfunctions including acute tubular necrosis, rejection, toxicity of immunodepressive preparations, renal artery stenosis, renal vein thrombosis and postbiopsy renal arteriovenous malformation are known to appear in the early postoperative period in patients with transplanted kidney. Acute transplant rejection usually occurs on day 4-5 after transplantation [5]. This situation makes working out of prognostic criteria of complication development in patients with transplanted kidney rather vital.

The aim of the investigation was to assess the reliability of predicting complications in the early postoperative period after kidney transplantation on the basis of the data obtained by monitoring redox potential in blood plasma.

Materials and Methods. 60 patients were examined in N.V. Sklifosofsky Research Institute of Emergency Care (Moscow) after kidney transplantation in the early postoperative period, which according to [6] lasted no more than 30 days. Two groups were formed: group 1 without complications in the postoperative period $(n=36)$ and group 2 with complications $(n=24)$. A total of 982 measurements (521 and 461 measurements in group 1 and 2 , respectively) were performed.

The study complies with the Declaration of Helsinki (the Declaration was passed in Helsinki, Finland, June, 1964, and revised in October, 2000, Edinburg, Scotland) and was performed following approval by the Ethic Committee of N.V. Sclifosovsky Research Institute of Emergency Care. Written informed consent was obtained from every patient.

The condition of the graft was evaluated basing on clinical observations and a wide spectrum of laboratory findings, as well as ultrasound examination and needle graft biopsy. Blood plasma was obtained by centrifugation of the whole blood, collected with the help of a vacuum system of blood specimen collection in the tubes with lithium heparin, for $15 \mathrm{~min}$ at $1,500 \mathrm{~g}$ using centrifuge $3.12 \mathrm{CR}$ (Jouan, France). The sample volume for examination amounted to $2 \mathrm{ml}$.

Redox potential measurements in blood plasma were performed using platinum electrode relative to a reference saturated silver chloride electrode for comparison. A platinum electrode was chosen as a working one, because its surface is covered by platinum oxide, the composition of which depends on the oxidation-reduction properties of the tested medium. Potentiostat IPC-Pro L (Cronas, Russia) was used for recording potential/time dependence. The time of registration was $15 \mathrm{~min}$. The electrode was electrochemically processed before each measurement [2].

Experimental data were analyzed using the software packages Statistica 6.0, EViews 8.0 (IHS Global, Inc.) and Visual Basic 6.0 IDE (Microsoft).

Results and Discussion. One of the important criteria of assessing patient's condition in the early postoperative period is prediction of complications, as they greatly influence the course of the disease and the results of treatment. Such prediction can be made in the form of estimating the probability of complications development as a function of statistical characteristics of clinical and laboratory parameters.

In the course of the previously conducted analysis of the mean RP values in the blood plasma substantial differences were found in the patients of the examined groups in the early postoperative period [7]. Comparison of the mean RP values dynamics in the examined groups in the course of monitoring showed, that the difference between the values reached $12 \mathrm{mV}$ already by day 5 , and $18 \mathrm{mV}$ by day 10 . And even on day 25 the difference in the mean RP values remains not less than $10 \mathrm{mV}$.

Taking into account the fact, that presence or absence of complications is a qualitative characteristic, a probit model was chosen to assess the complication occurrence probability/RP value relationship [8].

Probit analysis, being used in various fields, was applied for the first time in toxicology to evaluate the effect of the toxicant dose on the disease outcome. This is a statistical method for the analysis of binary variable dependence on numerous factors, using normal distribution. The probit model makes it possible to estimate the probability, that the analyzed (dependent) variable will take value "1" at the given values of factors (i.e. this is the assessment of the "one" proportion at a given value of factors).

Its basis is an interpretation of the function

$$
\Phi(x)=\frac{1}{\sqrt{2 \pi}} \int_{-\infty}^{x} e^{-t^{2 / 2}} d t
$$

of the standard normal distribution $\mathrm{N}(0,1)$ as a conventional probability of the event at a given value of 
its argument, by way of which linear transformation of the observed value is used.

Let's consider the sum $S$ of the linear function of $E$ with undetermined coefficients $\alpha$ and $\beta$, and of an accidental error $U$, having normal distribution $\mathrm{N}(0,1)$, i.e.

$$
S=\alpha+\beta E+U \text {. }
$$

The value $S$ possesses the following property $P$ :

$$
\begin{aligned}
P(S>0 / E & =e)=P(\alpha+\beta e+U>0)=P[U>-(\alpha+\beta e)]= \\
& =1-\Phi[-(\alpha+\beta e)]=\Phi(\alpha+\beta e) .
\end{aligned}
$$

If we assume, that the value of $S>0$ is operation failure, then coefficients $\alpha$ and $\beta$ may be estimated on the basis of the sample data using the method of maximum likelihood. Log likelihood function may be presented in the form of:

$$
I(\alpha, \beta)=\sum_{i: S>0} \ln \left[\Phi\left(\alpha+\beta \cdot e_{i}\right)\right]+\sum_{i: S<0} \ln \left[1-\Phi\left(\alpha+\beta \cdot e_{i}\right)\right],
$$

where $e_{i}$ is the value of RP of $i$-patient.
Maximization of this function by unknown parameters $\alpha$ and $\beta$ makes it possible to estimate the parameters. The quality of the obtained model of probit analysis was evaluated with the help of analogs of the coefficient of determination $R^{2}$ for the ordinary linear regression, namely, coefficients of determination:

$$
\text { MacFadden } R^{2}=1-\frac{L_{1}}{L_{0}} \text { and pseudo } R^{2}=1-\frac{1}{1+2\left(L_{1}-L_{0}\right) / n} \text {, }
$$

where $L_{1}$ is the value of maximum $/(\alpha, \beta)$ in both variables, and $L_{0}$ is the maximum value of the logarithmic likelihood function for a trivial model, i.e. $I(\alpha, 0)[9]$.

One of the important tasks of the present investigation was to analyze the experimental data in order to test their fit for the model of the normal probability distribution. It was a major requisite for the correct application of probit analysis. Because of the small sample volumes

\begin{tabular}{|c|c|c|c|c|c|c|c|c|}
\hline \multirow{2}{*}{ Days } & \multicolumn{4}{|c|}{ Group without complications } & \multicolumn{4}{|c|}{ Group with complications } \\
\hline & n & d & $g_{1}$ & $\mathbf{b}_{2}$ & $\mathrm{n}$ & d & $g_{1}$ & $\mathbf{b}_{2}$ \\
\hline 1 & 21 & 0.7570 & -0.1854 & 2.6099 & 9 & 0.7483 & 0.1954 & 2.9341 \\
\hline 2 & 16 & 0.8420 & 0.1116 & 2.1206 & 8 & 0.7656 & 0.0771 & 2.9196 \\
\hline 3 & 13 & 0.7847 & 0.2844 & 2.3128 & 7 & 0.8466 & 0.0936 & 1.8910 \\
\hline 4 & 12 & 0.8715 & -0.1837 & 1.8635 & 7 & 0.8796 & 0.2361 & 1.7591 \\
\hline 5 & 14 & 0.8248 & 0.0150 & 2.2998 & 10 & 0.7170 & -0.0413 & 3.6655 \\
\hline 6 & 15 & 0.8778 & 0.1130 & 1.7365 & 14 & 0.8169 & -0.0820 & 2.2758 \\
\hline 7 & 21 & 0.8496 & 0.1672 & 1.9266 & 14 & 0.7372 & -0.2410 & 4.0035 \\
\hline 8 & 25 & 0.8180 & -0.4110 & 2.5019 & 20 & 0.7236 & -0.1613 & 4.1224 \\
\hline 9 & 24 & 0.7930 & -0.7701 & 3.7151 & 17 & 0.7863 & -0.4123 & 3.0752 \\
\hline 10 & 22 & 0.7870 & -0.6938 & 2.7733 & 19 & 0.8373 & -0.2530 & 2.1508 \\
\hline 11 & 17 & 0.8056 & 0.2081 & 2.2472 & 16 & 0.8523 & 0.3656 & 1.9621 \\
\hline 12 & 18 & 0.8202 & -0.3295 & 2.4292 & $13^{5}$ & 0.9047 & -0.1297 & 1.6779 \\
\hline 13 & 21 & 0.8064 & -0.5267 & 2.9883 & $16^{1,2,3,4}$ & 0.7373 & -1.4769 & 5.7205 \\
\hline 14 & 25 & 0.7993 & 0.1794 & 2.8546 & $15^{1,3,4}$ & 0.7809 & -1.0541 & 3.9114 \\
\hline 15 & 28 & 0.7700 & -0.7406 & 3.4350 & $21^{1,3,4}$ & 0.7568 & -0.7760 & 3.0246 \\
\hline 16 & 24 & 0.7496 & 0.0439 & 2.8017 & $17^{1,2,3,4}$ & 0.7077 & -1.2388 & 5.5729 \\
\hline 17 & 18 & 0.7926 & -0.5587 & 2.6149 & $17^{1,2,3,4}$ & 0.7087 & -1.5177 & 5.8987 \\
\hline 18 & 15 & 0.8446 & 0.2212 & 2.0916 & 14 & 0.8850 & -0.3068 & 1.7385 \\
\hline 19 & 17 & 0.8571 & 0.4793 & 2.2698 & $9^{3}$ & 0.9186 & -0.1221 & 1.4754 \\
\hline 20 & $21^{3,5}$ & 0.8843 & -0.1335 & 1.7701 & 12 & 0.7985 & -0.5842 & 2.4928 \\
\hline 21 & 24 & 0.8301 & -0.1100 & 2.0264 & 10 & 0.8623 & -0.5949 & 2.5278 \\
\hline 22 & 21 & 0.7938 & -0.6166 & 2.9776 & 16 & 0.7957 & -0.6675 & 2.6158 \\
\hline 23 & 16 & 0.8142 & 0.0261 & 1.9599 & 14 & 0.7635 & -0.5272 & 3.1462 \\
\hline 24 & 7 & 0.7810 & -0.1197 & 2.5679 & 9 & 0.8246 & 0.2861 & 2.0881 \\
\hline 25 & 9 & 0.8687 & 0.3444 & 1.6889 & 9 & 0.8624 & -0.1695 & 1.7779 \\
\hline
\end{tabular}
typical for clinical investigations, a reliable estimation of the distribution density by the available data is rather

Comparative dynamics of the redox potential mean values by days and some sampling characteristics in the groups with and without complications

$\mathrm{N}$ o t e. For the values printed in bold type, a statistically significant $(\mathrm{p}<0.05)$ difference of the sample distribution from normal was obtained according to the tests: ${ }^{1}$ for symmetry; ${ }^{2}$ for excess; ${ }^{3}$ ShapiroWilk test; ${ }^{4}$ Epps-Pally criterion; ${ }^{5}$ for normalized median absolute deviation. Here $n$ is sample size; $d=\frac{1}{n s^{*}} \sum\left|x_{i}-\bar{x}\right|$ is sample normalized median absolute deviation; $g_{1}=\frac{1}{n\left(s^{*}\right)^{3}} \sum\left(x_{i}-\bar{x}\right)^{3}$ is sample asymmetry coefficient; $b_{2}=\frac{1}{n\left(s^{*}\right)^{4}} \sum\left(x_{i}-\bar{x}\right)^{4}$ is sample coefficient of excess; $\left(s^{*}\right)^{2}=\frac{1}{n} \sum\left(x_{i}-\bar{x}\right)^{2}$ is sample variance. 
problematic, the choice of normal distribution may be considered optimal in terms of the maximum entropy principle [10]. However, it is necessary to make certain, that such a choice is not disproved by the results of statistical criteria applied for testing normal distributions.

Testing the sample distribution deviation from the normal was performed in compliance with the standard [11]. Tests for symmetry, excess value, as well as Shapiro-Wilk test and Epps-Pally criteria were used by us [12, 13]. Additionally, the criterion based on the analysis of normalized median absolute deviation was also applied [14].

There are some difficulties in using this standard in practice, as not all included criteria are realized in the available statistical packages, and the tables of the threshold values, given in the standard, are incomplete, i.e. have blanks. Therefore, the value of Shapiro-Wilk criterion was calculated using Statistica program, whereas test criteria for symmetry, excess values and Epps-Pally criterion were estimated with the help of a specially devised auxiliary program code. Threshold values for the missing sample volumes of test criteria for symmetry, excess and normalized median absolute deviation were counted by interpolation, and for Epps-Pally criterion normalized transformation was used [15]. The results of testing the sample distributions for deviation from the normal law are presented in the Table.

As it is seen from the Table, in group 1 sample distributions successfully passed the test for normality for all days (except day 20) according to five criteria, four of which are included into the standard. As to group 2, deviations from the normal law, judging by the criteria, are fixed beginning only from day 12 . Thus, application of the normal law of probability for building prediction

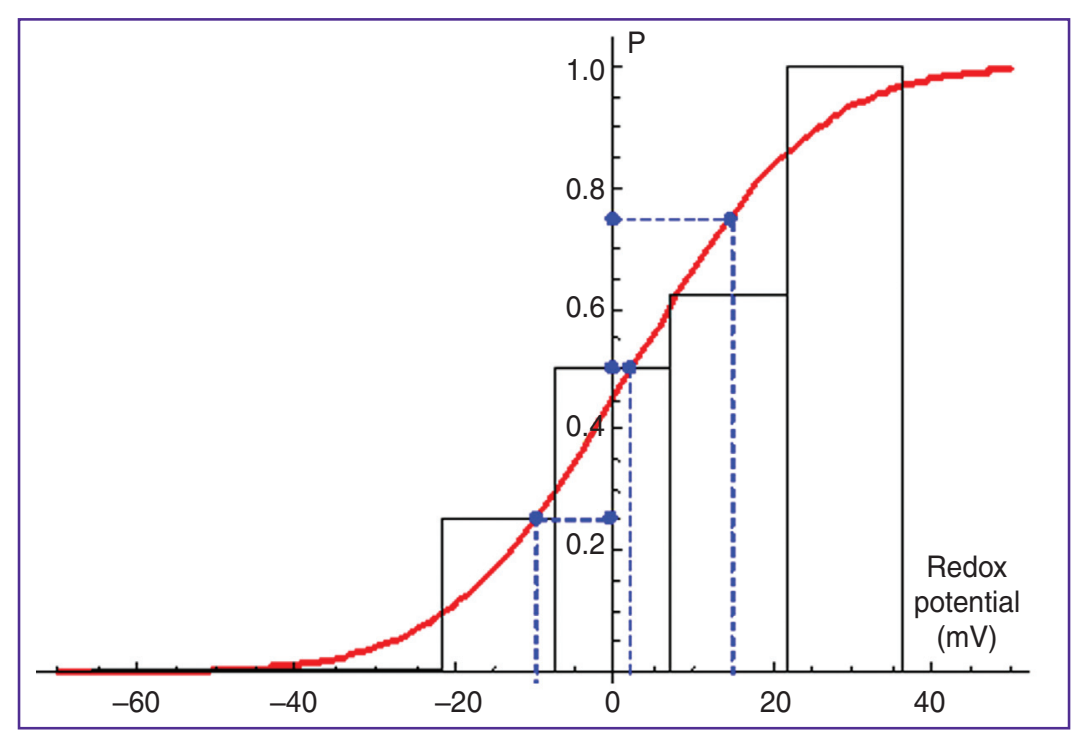

Figure 1. Approximation of dependence of conventional probability of complications on the value of redox potential in blood plasma on day 6 after the operation for kidney transplantation based on the sample data using a probit curve

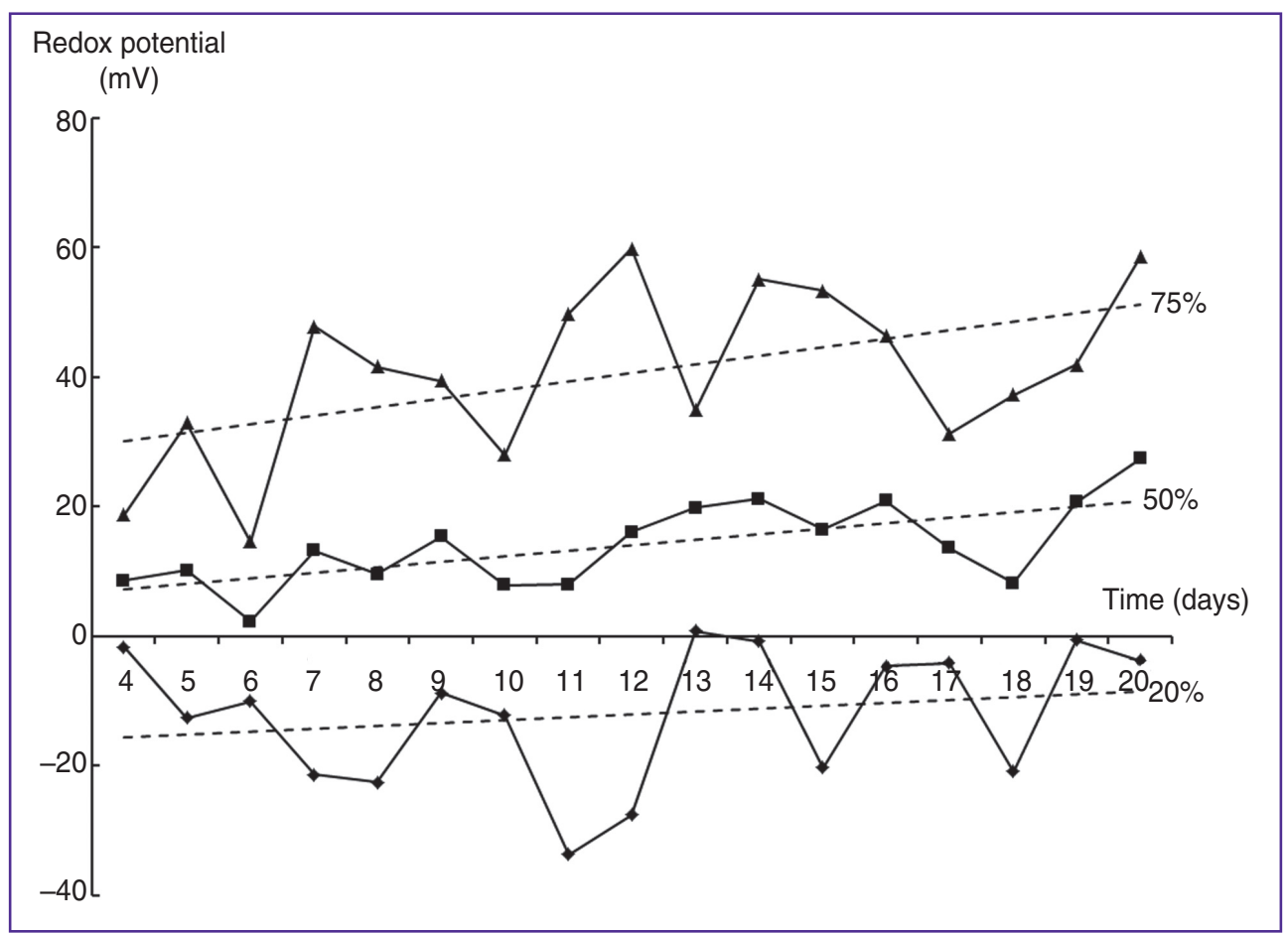

Figure 2. Dynamics of changes in the conventional probability of complications $(25,50,75 \%)$ depending on the time of postoperative monitoring 
models of complications is quite permissible in the early postoperative period, just when such prediction is very urgent.

Application of probit analysis enabled us to estimate the probability of complications depending on the $\mathrm{RP}$ value on the selected day of the postoperative period during patient's hospital stay. An example of graphic interpretation of the probability of complication development on the selected day is presented on Figure 1.

Thus, analyzing experimental data for the examined groups of patients with the help of probit analysis, values of conventional probability of complications depending on the day of postoperative monitoring have been estimated (Figure 2).

Conclusion. Graphic interpretation of the probability of complications on the selected day and the analysis of experimental data in patients after kidney transplantation using probit analysis enable urologists to determine conventional probability of complications depending on the day of the postoperative monitoring of the redox potential. A high probability of early complication prediction in patients with transplanted kidney using redox potential monitoring has been proved by our studies.

Study Funding. This work was supported by the grant of the Russian Scientific Fund (project No.14-29-00194); D. Mendeleyev University of Chemical Technology of Russia, Moscow.

Conflicts of Interest. There is no specific conflict related to the authors of this article.

\section{References}

1. Rael L.T., Bar-Or R., Mains C.W., Slone D.S., Levy A.S., Bar-Or D. Plasma oxidation-reduction potential and protein oxidation in traumatic brain injury. J Neurotrauma 2009; 26(8): 1203-1211, http://dx.doi.org/10.1089/neu.2008-0816.

2. Khubutiya M.Sh., Evseev A.K., Kolesnikov V.A., Goldin M.M., Davydov A.D., Volkov A.G., Stepanov A.A. Measurements of platinum electrode potential in blood and blood plasma and serum. Russian Journal of Electrochemistry 2010; 46(5): $537-541$, http://dx.doi.org/10.1134/s1023193510050071.

3. Goldin M.M. Volkov A.G., Khubutiya M.S.,
Kolesnikov V.A., Blanchard G.J., Evseev A.K., Goldin M.M., Teselkin Yu.O., Davydovh B.V. Redox potential measurement in aqueous solutions and biological media. ECS Transactions 2008; 11(21): 39-49, http://dx.doi.org/10.1149/1.2928905.

4. Pinchuk A.V., Aleksandrova I.V., Goldin M.M., Evseev A.K., Sorokin B.A. Assessment of the state and quality of treatment in posttransplant kidney patients, by monitoring the serum redox potential. Transplantologiya 2011; 2-3: 29-33.

5. Dubovsky E.V., Russell C.D., Erbas B. Radionuclide evaluation of renal transplants. Semin Nucl Med 1995; 25(1): 49-59, http://dx.doi.org/10.1016/s0001-2998(05)80006-6.

6. Ko G.-Y., Sung K.-B., Yoon H.-K., Lee S. Early posttransplantation portal vein stenosis following living donor liver transplantation: percutaneous transhepatic primary stent placement. Liver Transpl 2007; 13(4): 530-536, http://dx.doi. org/10.1002/lt.21068.

7. Goldin M.M., Khubutia M.Sh., Evseev A.K., Goldin M.M., Pinchuk A.V., Pervakova E.I., Tarabrin Y.A., Hall P.J. Noninvasive diagnosis of dysfunctions in patients after organ transplantation by monitoring the redox potential of blood serum. Transplantation 2015; 99(6): 1288-1292, http://dx.doi. org/10.1097/TP.0000000000000519.

8. Finney D.J. Probit analysis. Cambridge University Press; 2009; $272 \mathrm{p}$

9. Nosko V.P. Ekonometrika dlya nachinayushchikh (dopolnitel'nye glavy) [Econometrics for beginners (additional chapters)]. Moscow: IEPP; 2005; 379 p.

10. Tu Dzh., Gonsales R. Printsipy raspoznavaniya obrazov [Principles of image recognition]. Moscow: Mir; 1978; $412 \mathrm{p}$.

11. GOST $R$ ISO 5479-2002. Statisticheskie metody. Proverka otkloneniya raspredeleniya veroyatnostey ot normal'nogo raspredeleniya [GOST R ISO 5479-2002. Statistical methods. Testinig probability distribution variance from the normal distribution]. Moscow: Izd-vo standartov; 2002.

12. Kobzar' A.I. Prikladnaya matematicheskaya statistika [Applied mathematical statistics]. Moscow: Fizmatlit; 2008; 816 p.

13. Lemeshko B.Yu., Lemeshko S.B. Comparative analysis of criteria for testing probability distribution variance from the normal law. Metrologiya 2005; 2: 3-24.

14. Bol'shev L.N., Smirnov N.V. Tablitsy matematicheskoy statistiki [Mathematical statistics tables]. Moscow: Nauka; 1983; $416 \mathrm{p}$.

15. Gaydyshev I.P. Reshenie nauchnykh $i$ inzhenernykh zadach sredstvami Excel, VBA i $\mathrm{C} / \mathrm{C}++$ [Solution of scientific and engineering tasks using Excel, VBA и $\mathrm{C} / \mathrm{C}++]$. Saint Petersburg: BKhV-Peterburg; 2004; 504 p. 